\title{
Entdeckung der Alten Welt \\ Zum Funktionsgewinn früher Amerikareiseberichte in der barocken Kompilatorik
}

\section{Nicolas Detering}

\begin{abstract}
While some American travelogues developed a relativist mode of cultural description already in the $16^{\text {th }}$ century, it was not until the early $18^{\text {th }}$ century that it became fashionable to criticize the >civilized European < by comparing him with the >good savage $<$. The article peruses a number of $17^{\text {th }}$ century collections and compilations of earlier American travel books, and argues that they provide an important link between Humanism and the Early Enlightenment. In particular, in their selections they challenge the temporal attributions of new and old, thus enabling the association of America with nativeness and Europe with modernity.

Title: The Discovery of the Old World. Functions of Early American Travelogues in Baroque Compilations

Keywords: early American travelogues; cultural relativism; concepts of Europe; reception of Jean de Léry; compilation as genre
\end{abstract}

Seit der antiken Geographie war man überzeugt davon, in Europa auf dem zwar kleinsten, aber klimatisch angenehmsten, landschaftlich schönsten und überhaupt besten Erdteil zu leben - ein Gemeinplatz, der durch die militärische und ökonomische Expansion nach Asien und Amerika an Plausibilität noch gewann: Wie sonst ließ sich der exploratorische Erfolg der Europäer erklären, wie sonst ihre Fähigkeit, andere Kontinente zu kolonialisieren, wie sonst das göttliche Privileg der christlichen Offenbarung, das den >Wilden< in Amerika versagt geblieben war? So schrieb der spanische Rechtsgelehrte Juan López de Palacios Rubios 1513, dass Gott den amerikanischen Ureinwohnern sicher früher christliche Missionare geschickt hätte, wenn sie ein würdigeres Volk gewesen wären; schließlich habe er auch Augustinus von Canterbury nach England gesandt (vgl. Pagden 1993: 7f.). Ihre Fremderfahrungen suchten die Amerikareisenden auf der Grundlage der antiken Ethnographie (etwa der Plinianischen Naturgeschichte, in der auch von Monstren und Wilden berichtet wird) zu begreifen, und sie betrachteten die unchristlichen Riten der indigenen Bevölkerung meist mit Grauen. ${ }^{1}$ Zum Sinnbild einer unheimlichen Alterität erhob man den Kanni-

1 Zur Rezeption antiker Wissenschaften und Ethnographie in den Amerikareiseberichten siehe v.a. Grafton 1992 sowie Mason 1994. Zur Konstruktion der Dichotomie von ,Wilden und ZZivilisierten، siehe zuletzt Seth 2010 u. Kiening 2006 sowie die Stan-

ZiG | Zeitschrift für interkulturelle Germanistik 9|2018|H1 | @ transcript 2018 
balismus der >Wilden $<$ der den zivilisatorischen Primat der Europäer zweifelsfrei zu belegen schien und zum festen Bestandteil der europäischen Amerikaimagination wurde. ${ }^{2}$

Dennoch begegnet im ethnographischen Vergleich mancher Amerikaberichte - zu nennen wären Francisco Lopez de Gómaras Histoire générale des Indes (1552), André de Thevets Les Singularités de la France antarctique (1557) und José de Acostas Historia moral y natural de las Indias (1591) - auch eine europäische Selbstkritik, in der nicht mehr nur das Fremde, sondern auch das kulturell Eigene vermessen wurde. Als Resultat entwickelten sich in Frankreich gegen Ende des 16. Jahrhunderts sogar Frühformen eines kulturellen Relativismus. ${ }^{3}$ Oft hervorgehoben wurde in diesem Zusammenhang die brasilianische Reisebeschreibung des Hugenotten Jean de Léry, die 1578 unter dem Titel Histoire d'un voyage faict en la terre du Brésil, autrement dite Amérique erschien (vgl. Lestringant 1990: 47-83, Lestringant 1999, Frübis 2008). Léry vergleicht die brasilianischen >Wilden< durchgehend mit den Einwohnern der bekannten Welt: Die brasilianischen Tupinambá haben zwar keine richtige Vorstellung von Gott - aber sie gleichen darin nur den »plus qu'endiablez Atheistes dont la terre est maintenant toute couuerte par deçà «4 (Léry 1578: 265). Sie sind barbarisch und roh ihren Feinden gegenüber, aber sie sind dennoch fähig zur Vernunft und lassen sich von guten Argumenten überzeugen - »mieux que ne font la pluspart des paysans, voire que d'autres de par deçà qui pensent estre bien habiles «5 (ebd.: 290). Am deutlichsten wird Lérys normative Äquidistanz am Ende des Kapitels über den brasilianischen Kannibalismus, den er mit Lust an brutaler Ausführlichkeit über viele Seiten schildert, um sodann zur finalen Pointe anzusetzen: Der Leser möge nach all diesen Schrecklichkeiten aber nicht glauben, dass Anthropophagie allein eine amerikanische Unsitte sei! Man denke nur an Vergleichbares »par deçà«, wie die Wendung stets lautet: an die Wucherer und Zinsmacher zum Beispiel, welche die Witwen und Waisen gleichsam bei lebendigem Leibe verspeisen und grausamer noch seien als die Amerikaner, die ihre Opfer wenigstens

dardwerke von Lestringant 1990 u. Bitterli 1976. Zu den deutschsprachigen Amerikareiseberichten vgl. Harbsmeier 1994 u. Neuber 1991.

2 | Zum Motiv des Kannibalen in der Frühen Neuzeit vgl. zuletzt Watson 2015, Arend 2006 sowie Holdenried 2004.

3 | Mit ,Relativismus، ist hier nicht die moderne Variante gemeint, der zufolge eine objektive Erkenntnis der Welt oder universale Werturteile überhaupt unmöglich seien, sondern lediglich die (ziemlich alte) Vorstellung, dass Normen historisch, sozial und kulturell geprägt sein können. Zur konzeptuellen Unterscheidung der verschiedenen Alternativen zum Universalismus vgl. Berlin 1991, der allerdings dafür plädiert, für ältere Auffassungen (Montaigne, Vico, Herder) nicht 'Relativismus`, sondern den Begriff pluralism zu verwenden.

4 | "[M]ehr-als-besessene Atheisten, von denen die Erde bei uns ganz übersät ist“ (Übers. N.D.).

5 | "[B]esser als die meisten Bauern, ja auch als andere bei uns, die sich für sehr geschickt halten" (Übers. N.D.). 
töten, bevor sie sie verschlingen. Die Verfolgung der Hugenotten, die Vergleiche des brasilianischen Kannibalismus mit der katholischen Eucharistie, die Pariser Modesucht als Zivilisationskrankheit, der um sich greifende Atheismus das alles beschreibt Léry als Symptome einer moralischen Depravation, die den >wilden< Ritus der Tupinambá relativieren und die Erklärungsbedürftigkeit des Eigenen in den Vordergrund rücken.

Diese Ansätze eines relativistischen Kulturvergleichs wirkten zwar deutlich auf zwei Essais Montaignes (1580), nämlich Des Cannibales (I,31) und Des Coches (III,6), ${ }^{6}$ blieben im 16. Jahrhundert dennoch die Ausnahme. ${ }^{7}$ Überhaupt ist die Wirkung der ethnographischen Amerikareiseberichte auf den Humanismus umstritten. Die Forschung hat ermittelt, dass in Frankreich zwischen 1480 und 1609 viermal so viele Bücher über Asien und die Türken erschienen wie über Amerika (vgl. Atkinson 1935). ${ }^{8}$ Gerade im deutschen Sprachraum konnte die Selbstbespiegelung der französischen Reisebeschreibungen zunächst nur zögerlich Fuß fassen. Obwohl das ökonomische Engagement der Welser im heutigen Venezuela in der ersten Hälfte des 16. Jahrhunderts zu einer breiten deutschen Rezeption von Americana geführt hatte (vgl. Neuber 1991: 222f.), bleiben vereinzelte Rezeptionsspuren bei Sebastian Brant und Johann Fischart eher unspezifisch. Ihre epochale Wirkung entfalteten die Reiseberichte daher wohl weniger in der Renaissance als vielmehr mit der Dynamisierung des europäischen Selbstbewusstseins um 1700.9 » Lire les récits de voyages, ce fut s'évader; ce fut passer de la stabilité d'esprit au mouvement«, formuliert schon Paul Hazard, »[c]e fut une des raisons pour lesquelles la conscience de la vieille Europe se troubla, et, voulant être bouleversée, le fut « ${ }^{10}$ (Hazard 1935: 28f.). Die frühen Amerikaschrif-

6 | Zum Einfluss Thevets und Lérys auf Montaignes Des Cannibales siehe Enders 1993, Lestringant 1990: 133-149, und Weinberg 1968. Zu Des Coches vgl. zuletzt eingehend Hampton 1997. - Kohl 1983: 22f., bestreitet, dass es sich bei Montaignes "Kritik an den Voreingenommenheiten seiner Zeit" um eine Form des Kulturrelativismus handele. Die "Projektionsbestimmtheit" seiner Sichtweise trete deutlich zutage "in den konkreten Einzelzügen seiner positiven Bestimmung der brasilianischen Wilden".

7 | Neben den genannten ließen sich als Belege für eine positive, bisweilen idealisierende Darstellung der amerikanischen Völker noch Marc Lescarbots Histoire de la Nouvelle France (1609), Gabriel Sagards Histoire du Canada et des voyages que les Frères Mineurs Recollects y ont faicts (1636) und Jean Baptiste Du Tertres Histoire generale des Antilles habitées par les François (1667) anführen; siehe Kohl 1983: 30f.

8 | Elliott (1970: 12) vermutet, Ähnliches gelte für England und Italien: "[T]here is little sign of literary interest before the 1550s, when the new Anglo-Spanish connection provided a belated stimulus". Auch Enders (1993: 196) hält fest, "daß Amerika im geistigen Leben Frankreichs während des 16. Jahrhunderts keine besondere Rolle gespielt hat". Weitere Literaturhinweise zu dieser Frage bei Mason 1994: $141 \mathrm{f}$.

9 | Zur ,Kulturalisierung, des Europadenkens durch die Konfrontation mit utopistischen, exotistischen und primitivistischen Fremdbildern um 1700 siehe Detering 2017: 464-483.

10 | "Die Reiseberichte zu lesen, das hieß entfliehen; das hieß, von der Stabilität des Geistes zur Bewegung überzugehen [...]. Das war einer der Gründe, weshalb das Be- 
ten boten späteren Interpreten eine empirische Grundlage für deren Kulturvergleiche, insbesondere in der Früh- und Hochaufklärung des 18. Jahrhunderts.

Die Forschung hat diese Transformationsverspätung verschiedentlich festgestellt und die Rezeption französischer Reiseberichte etwa bei John Locke untersucht (vgl. Talbot 2010). Für den deutschen Kontext aber unterschätzt man noch immer die Mittlerrolle, welche die Kompilationsliteratur des Barock einnahm. Sie wählte markante Episoden aus den Amerikabeschreibungen aus, kommentierte sie und reduzierte die Vielzahl neuer Informationen auf besonders >curieuse< Aspekte, die dadurch kanonisiert und popularisiert wurden. Wie ich erstens anhand der Reihenedition aus dem De-Bry-Verlag, zweitens an einem >Amerikanischen Kalender<, drittens an einem gelehrt-systematischen Florilegium, viertens am Beispiel der >bunten < Dialogkompendien Erasmus Franciscis zeigen möchte, pointieren die barocken Kompilatoren das Wechselspiel von europäischer Selbstüberhöhung und Selbstkritik, das bei Léry und anderen angelegt war. Das geschieht insbesondere, so im Folgenden die leitende Hypothese, indem sie die räumlich-ethnische Differenz zwischen Europa und Amerika verzeitlichen und kulturelle Fremdheit mit historischer Distanz verknüpfen. Damit deutet sich im barocken Sammelschrifttum eine Reflexion europäischer Lebensformen im vermeintlich Vor- und Frühgeschichtlichen an, die, wie ich fünftens ausblickend andeute, in der Frühaufklärung expliziert und geschichtsphilosophisch entwickelt werden sollte.

\section{Antikisierte Menschenfresser? Die Kupferstiche ZU de Brys Neuedition VON Stadens WahrHaftiger Historia (1593)}

Der calvinistische Goldschmied und Kupferstecher Theodor de Bry begann 1590 eine Serie von älteren Amerikaberichten herauszugeben, die heute unter den Titeln West-Indische Reisen oder Reisen in das occidentalische Indien bekannt sind. ${ }^{11}$ Bis 1634 wurde die Reihenedition von seinen Söhnen fortgesetzt. Wenngleich die de Brys bei der Selektion französischer, italienischer, spanischer und deutscher Reiseberichte unsystematisch und gelegentlich mit konfessioneller Parteilichkeit vorgingen - die katholischen Berichte sind deutlich in der Minderzahl -, waren die Übersetzungen ins Deutsche und Lateinische doch so verdienstvoll und die Auflagen so hoch, dass man noch im 18. Jahrhundert auf die Serie zurückgreifen konnte. Ihr Erfolg dürfte nicht nur das europäische Bild der indigenen Amerikaner wesentlich mitgeprägt haben, sondern erklärt auch die Prädominanz französisch-hugenottischer Reiseberichte im 17. Jahrhundert (vg1. Lestringant 1990: 279-311).

wusstsein des alten Europas sich beunruhigte, und da es erschüttert sein wollte, wurde es das" (Übers. N.D.).

11 | Zu der Serienedition des De-Bry-Verlags siehe die neueren Studien von Perplies 2017, Groesen 2008 u. Greve 2004. 
Als wirkmächtig erwiesen sich insbesondere die hochwertigen Kupferstichillustrationen aus der Werkstatt de Bry. Augenfällig ist dabei die Tendenz einer klassizistischen Nostrifizierung der Körperdarstellung. Die scheinbare Nacktheit der >Indianer< begünstigte die Analogiebildung zur Ikonographie antiker Statuen, mit der man den unbekleideten menschlichen Körper spätestens seit der Renaissance zwingend verband. Das zeigt sich unter anderem im dritten Band der De-Bry-Reihe, der Neuedition des wohl wichtigsten deutschsprachigen Amerikaberichts, nämlich Hans Stadens Warhaftiger Historia und beschreibung eyner Landtschafft der Wilden Nacketen, Grimmigen Menschfresser-Leuthen in der Newenwelt America, erschienen zuerst 1557. Vergleicht man die schematischen und grobflächigen Holzschnitte der Erstausgabe mit den kunstvollen Kupfern der De-Bry-Edition von 1593 (Abb. 1 und 2), sticht zunächst die anatomisch genauere Konturierung der Muskelpartien ins Auge. Die statuarische Heroenstellung der Kriegerfiguren am linken Bildrand sowie die formalisierte Herrschaftsgeste des in die Hüfte gestemmten Arms der im Zentrum stehenden Figur entstammen der antiken bzw. der Renaissance-Ikonographie. Sie suggerieren eine Universalität von Gestik und Mimik, die mit den befremdlichen Sitten, die in Stadens Bericht beschrieben werden, durchaus kontrastiert.

Abbildungen 1 und 2: Holzschnitt- (links, Staden 1557: fol. $x j^{\nu}$ ) und Kupferstichillustration (Staden/Léry 1593: 37).
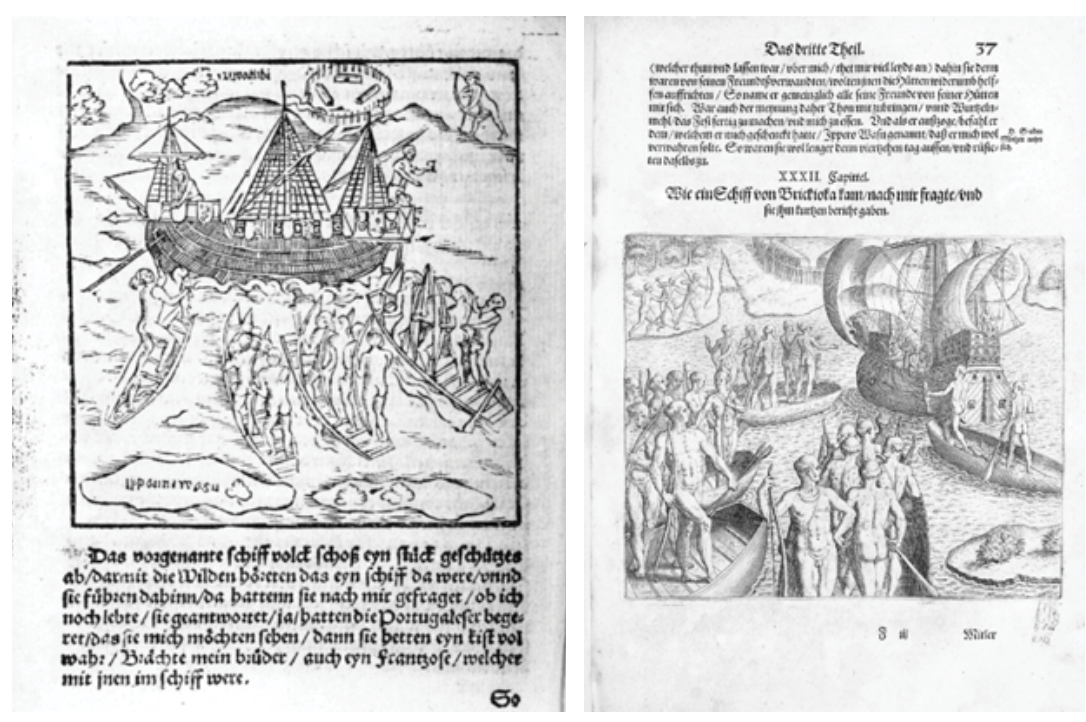

Bereits de Brys Frontispiz des Staden-Bandes (Abb. 3) überblendet ästhetische Vertrautheit und die Ostentation moralischer Fremdheit, wie sie sich insbesondere an dem brasilianischen Kannibalismus entspann. ${ }^{12}$ Die Figuren des Mittelteils

12 | Zu den Frontispizen und ihrer architektonischen Gestaltung siehe Christadler 2004. 
Abbildung 3: Kupferstich von Theodor de Bry (Frontispiz aus Staden/Léry 1593).

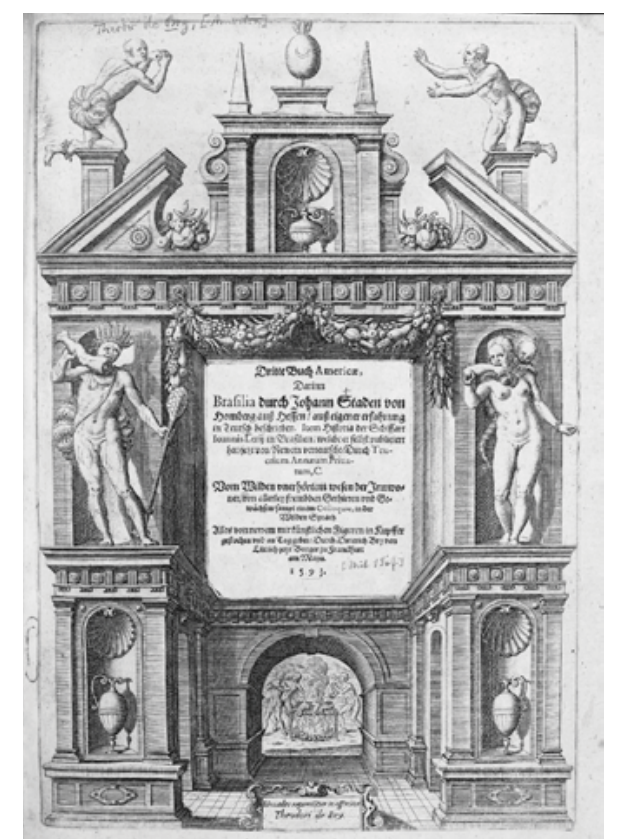

werden beim Verspeisen menschlicher Körperglieder gezeigt - und dabei selbst von einem Dämon angefallen. Zugleich aber integriert de Bry sie in die Blendnischen eines europäischen Architekturensembles, wo sie die Position antiker Statuen in Aedikulae einnehmen. Die Voluten, das Muschelwerk, die Ziervasen und die Floralarrangements sind zwar keine klassizistischen Ornamente, entsprechen aber dem ästhetischen Geschmack der Zeit und heben die Alterität brasilianischer Sitten umso stärker hervor. Die zivilisatorische Norm, die in der antithetischen Ordnung der vertrauten Bauelemente zum Ausdruck kommt, unterstreicht somit das transgressive Moment der amerikanischen Anthropophagie. Das kontrastive Zurschaustellen von Alterität ist aber nur ein Aspekt des Frontispizes. In der Haltung erinnern die beiden Kannibalen nämlich an Adam-und-Eva-Darstellungen, etwa auf Dürers bekanntem Kupferstich von 1504 (Abb. 4 und 5) - wie dort steht der amerikanische Mann zur Linken, die Frau zur Rechten; beide wenden den Kopf einander, den Oberkörper dem Betrachter zu; beide sind in Kontrapoststellung mit gespiegeltem Spielbein und geöffneten Armen dargestellt. Mit etwas Phantasie entspricht der Schlange auf Dürers Stich der schlangengeschwänzte Dämon, der sich bei de Bry um die Tupinambáfrau windet. 
Abbildungen 4 und 5: Bildausschnitte aus Abb. 3 (links). Albrecht Dürer: Der Sündenfall, Kupferstich, 1504 (Strauss 1980: 9).
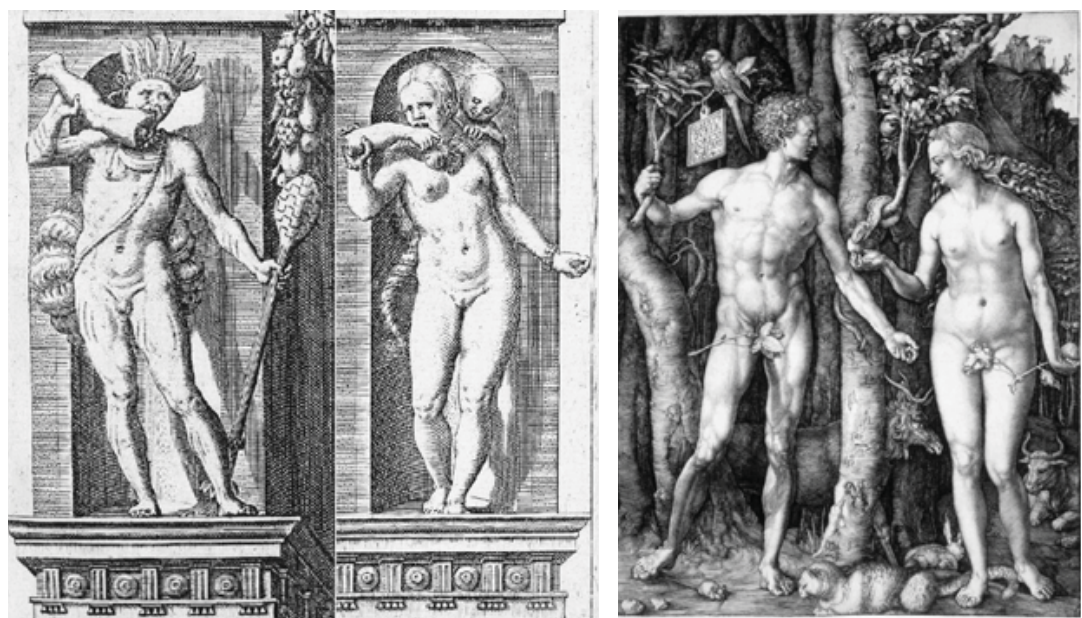

De Bry pervertiert das Paradiesmotiv zwar, indem er seine >Indianer $<$ menschliche Körper verschlingen lässt. Dennoch beerbt er eine Assoziation, die seit den frühesten Amerikareiseberichten topisch war: Bereits Vespucci hatte 1502 die nordbrasilianische Landschaft in seinem Mundus novus-Brief mit dem Garten Eden verglichen. ${ }^{13}$ Dass die Amerikaner niemals krank würden, dass sie keine Körperscham kennten und dass die Frauen keine Geburtsschmerzen empfänden, galt den französischen, spanischen und deutschen Reiseberichten als Zeichen paradiesischer Unschuld. Staden beispielsweise rechtfertigt die Nacktheit der >Wilden < damit, »dass der grosse Pracht vnd das Schmincken / die gemachte Haar / vnd gekreusselte Locken / die schweiffende / gefaltene Kleidung / köstlicher Gewand [...] vnd anderß dergleichen / darmit sich vnsere Weiber so meisterlich verstellen / viel mehr Vbels vnd Vnrahts anstiffte / denn die nackende blosse Wilden Weiber« (Staden/Léry 1593: 146). In der Bibel lese man schließlich, »daß sich Adam vnd Eua nach dem Fall / da sie nackend befanden / schämeten « (ebd.); weil die >Wilden< beim Anblick nackter Körper keine Wollust oder Scham empfänden, so die Implikation, entsprechen sie in diesem Punkt dem Dasein der ersten Menschen vor dem Sündenfall - freilich nur in diesem Punkt, denn weit öfter stellt Staden die Grausamkeit und Kriegslust der Tumpinambá heraus, denen er mehrmals zum Opfer zu werden droht.

Wie in Stadens Reisebeschreibung selbst konvergiert auch auf de Brys Frontispiz die kulturelle Abscheu vor den Menschenfressern mit einem historischen Fernweh nach Ursprünglichkeit, das sich in der Adam-und-Eva-Allusion ausdrückt: Die brasilianische Lebensweise wird abgewertet, aber ihre Andersartig-

13 Auch die Illustrationen zum Mundus novus erinnern bisweilen an Adam und Eva, wie Neuber (1991: 238) zeigt. Zum Motiv des Sündenfalls in anderen Illustrationen der De-Bry-Serie vgl. Greve 2004: 107-109. 
keit - vor allem ihre Nacktheit - assoziiert zugleich die verlorene Vergangenheit des Garten Edens. Vor dieser Folie wird das Eigene gleich doppelt sichtbar: als kulturell Europäisches und historisch Neuzeitliches. Wie zu zeigen sein wird, wurzelt in diesen Geschichtsentwürfen eine eurozentrische Weltwahrnehmung, die trotz ihrer vordergründigen Zivilisationskritik vor allem eines bewirkt, nämlich Differenz zu stabilisieren, um Europa als Prozessmodell zu installieren, »an dem die Geschichten und sozialen Formationen aller Gesellschaften gemessen und bewertet werden können« (Conrad/Randeria 2002: 14).

\section{JäHRLICH MerkWÜrdiges: AMERIKAWISSEN IN DER KaLENDERLITERATUR}

Die Legitimierung weltlicher Curiositas hatte seit dem Späthumanismus zur Ausbreitung kompilatorischer Formate geführt. ${ }^{14}$ Ließ die Wissbegierde vormals auf lasterhafte Hybris schließen, konnte man nun gerade den >neuen< Kontinent als merk-würdig vermarkten, nämlich im Wortsinne als >würdiger < Gegenstand geselliger Konversation und Ausdruck von Weltgewandtheit. »Frembder Leute frembder Sitten zu betrachten stehet schön«, lautet das Motto eines Americanischen Schiff-fahrten [...] Calenders (1675), »[d]aß man weiß zu discuriren / wie da pflegen her zu gehen« (Itzapolka 1675: fol. $4^{\mathrm{r}}$ ). Gestützt durch den Erfolg der De-Bry-Reiheneditionen um 1600 fanden die Americana einige Jahrzehnte später Eingang in die verschiedenen Kanäle der kompilatorischen Literatur, wo die ethnographischen >Altbestände< reorganisiert und auf Unterhaltsames verengt wurden. Unter ihnen war der Kalender ein besonders kräftiger, weil alltagswirksamer Rezeptionskatalysator. In der Mitte des 17. Jahrhunderts bürgerte sich die Mode ein, in die Kalender kleinere Erzählungen und kuriose Meldungen zu integrieren, gleichsam als kurzweilige Beigabe zum Ernst der astrologischen Praxis. So verfasste Hans Jakob Christoffel von Grimmelshausen von 1670 bis 1672 für den Nürnberger Verlag Felsecker einen Europäischen Wundergeschichten Calender, der neben dem nummerierten Wochen- und Tagesverzeichnis alter wie neuer Kalenderordnung sowie der Wetterprognose nach Planetenkonstellation auch eine Spalte mit simplicianischen Schelmenstücken bietet (vgl. Grimmelshausen 2009). ${ }^{15}$ Ebenfalls in Nürnberg erschien seit 1673 ein Cosmographischer Calender, der Felseckers Erfolgsmodell satztypisch nachempfunden ist, aber statt mit einer fiktionalen Erzählung mit »einer Summa« »von jedem Theil der Welt « aufwartet: In der ersten Spalte beschreibt er die Länder, Städte, Sitten und »höchst-wunderlichen Raritäten« Amerikas, in der nächsten diejenigen Asiens und in der dritten Afrikas (Stöffler 1673). Die >Praktik<, ein astrologischer Anhang, bietet einen Abriss Europas, das nicht nur auf diese Weise hervorgehoben wird: Das Titelkupfer, ein Nachstich von Joachim Sandrarts Frontispiz zu Mat-

14 | Vgl. zur Aufwertung der Neugierde im 17. und 18. Jahrhundert Blumenberg 1966: 201-433, und Kenny 2004.

15 | Zur Gattung der Kalenderliteratur vgl. Böning 2011. 
thäus Merians Neuwer Archontologia Cosmica (d'Avity 1638; siehe Wüthrich 1993: Abb. 176), zeigt die personifizierte Europa als wehrhafte Königin der Welt auf einem Sockel. Ihr zu Füßen stehen die halbnackten, damit zivilisatorisch herabgewürdigten Personifikationen Asiens und Amerikas, in deren Mitte Afrika auf einem Krokodil sitzt (Abb. 6).
Abbildung 6: Die vier Erdteile - Kupferstich
von Sigmund Gabriel Hipschmann nach einer Vorlage von Joachim Sandrart
(Frontispiz aus Stöffler 1673).

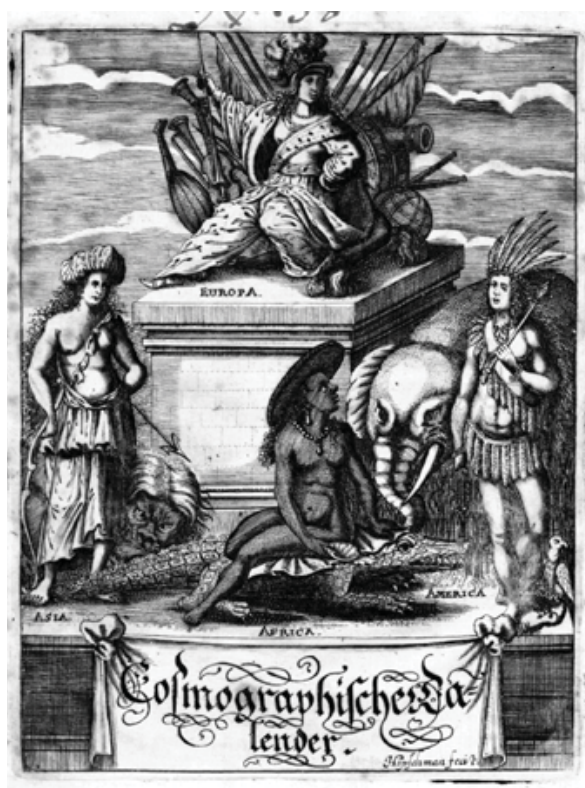

Hatte dieser >europhorischeく Kalender noch alle Teile der Welt berücksichtigt, gesellte sich ihm ${ }_{1675}$ der bereits zitierte Americanische Schiff-fahrten/Sitten-/Trachten-/Grausamkeiten- und Landschafften-Calender hinzu, der mindestens bis 1678 bestand. Während sein Frontispiz - zwei >Indianer< halten ein Bildnis Karls V., im Hintergrund sind gegen Hunde kämpfende >Wilde $<$ zu sehen $^{16}$ - wie im Vorläufer von dem Nürnberger Kupferstecher Sigmund Gabriel Hipschmann stammt, ist die Autorschaft bzw. die Redaktion des amerikanischen Ablegers ungewiss. Sicher ein Pseudonym ist der Name »Coran Itzapolka / geborne[r] Americaner, aus Quito«, der auf dem Titelblatt des ersten Jahrgangs erscheint und mit dem noch die Vorrede des zweiten Jahrgangs unterzeichnet ist. Wahrscheinlich steht er für den seit dem zweiten Jahrgang angegebenen Astrologen

16 | Das Motiv der Combat des Sauvages war seit dem 16. Jahrhundert topisch und wurde unter anderem in den Illustrationen zu Lérys und André Thevets Reiseschilderungen gestaltet (vgl. Frübis 2008: 195-197). 
Peter Freytag, über den sich allerdings kaum etwas eruieren lässt. In der ersten Zusatzspalte des Kalenders, erklärt die Vorrede, wird das Leben Christopher Kolumbus' erzählt, der »erst[e] Erfinder dieser neuen Amerikanischen Welt«; im zweiten werden die Landschaften und Sitten, im dritten Feld der »Götzendienst« der amerikanischen Einwohner behandelt, wobei der Kalender sich zunächst auf Peru beschränkt (Itzapolka 1675). Ganz weitgehend zitiert >Itzapolka< bzw. Peter Freytag aus Olfert Dapperts Bearbeitung von Arnoldus Montanus' De Nieuwe en Onbekende Weereld (1671), die 1673 in deutscher Sprache erschienen war. Beispielsweise vermerkt Freytag im dritten Teil seines amerikanischen Kalenders (1677), die Peruaner würden drei Götzenbilder der Sonne und des Donners anbeten, woraus der » Jesuit Acosta« geschlossen habe, »daß der Teuffel den Peruern [...] das Geheimniß des heiligen Nachtmahls unsers Heylandes / als auch der heiligen Dreyeinigkeit« (Freytag 1677: o.S.) eingegeben habe. Die Stelle ist wörtlich dem vier Jahre zuvor erschienenen Kompendium des Montanus entnommen, wo sie der Rubrik >Peruische Feste < zugeteilt ist. ${ }^{17}$ Wahrscheinlich hat Montanus aus einer niederländischen Übersetzung von Acostas Historia natural y moral de las Indias (1591) zitiert, die Jan Huygen van Linschoten 1598 veröffentlichte (d.i. Acosta 1598). Diese Übertragung wiederum war 1601 auch als neunter Teil der De-Bry-Reihenedition erschienen. Dort lautet die zitierte Stelle, »der Teuffel« habe »seinem Affenspiel nach / die Dreyfaltigkeit eyngeführet« (Acosta/Jansz 16o1: 233). Der Fall ist ganz typisch für das barocke Extraktionsverfahren: Die Kompilationen durchforsten Acostas jahrzehntealte Historia mit kosmographischer Neugierde auf Sachinformationen und Wertungen, sortieren diese neu, variieren sie leicht oder wiederholen sie in gleichem Kontext und lassen sie dadurch eingehen in populäre Medien wie den periodischen Kalender.

\section{Katechismen des Amerikawissens: Amerikagenealogie und Selbstkritik im gelehrten Florilegium}

Itzapolka und Freytag ordnen ihre Notizen nur vage und verzichten weitgehend auf eigenständige Deutung. Anders verfahren gelehrte Florilegien, welche die frühen Amerikaberichte bereits katechisieren. So publizierte der Oldenburger Hofhistoriograph Johann Justus Winckelmanns mit seiner Americanischen Neuen Welt Beschreibung (1664) eine gekürzte Fassung von Hans Stadens Historia und schickte ihr eine Reihe von »nachdenklichen Fragen« voraus, zu deren Be-

17 | Montanus/Dapper 1673: 350f.: "Die drey Götzenbilder der Sonne nenneten die Peruer Apointi, Cheriunti, und Intiquaoqui, welches so viel heisset / als Vater Sonne / Sohn Sonne / und Bruder Sonne. Auf gleiche weise benahmeten sie die drey Götzenbilder des Donners Chuquilla, Catuilla, und Intillapa. Der Jesuiet [sic] Akosta schliesset hieraus; daß der Teufel den Peruern hierdurch das Geheimnis des heyligen Nachtmahls unsers Heylandes / als auch der heyligen Drey-einigkeit gleichsam geoffenbahret". - Ebenfalls wörtlich zitiert einige Jahre später Eberhard Werner Happel die gleiche Stelle (vgl. Happel 1688: 1082). 
antwortung er die Reiseberichte anderer Autoren wie Vespucci, Acosta, Benzoni und Léry, aber auch Kosmographien und Traktate heranzog. Systematisierungsanspruch erhebt schon das »Ordnungs-Register der Capiteln« (Winckelmann 1664: fol. iiijf.), das dem Leser alle wichtigen Aspekte des Themas auf einen Blick präsentiert; für Übersichtlichkeit sorgen zudem die Marginalscholien und Maniculae, die eine rasche Orientierung ermöglichen, sowie schließlich ein mehrseitiges »Register Der Vornemsten Namen und Sachen « (ebd.: fol. gg jij ${ }^{\mathrm{r}}$ hh iiij ${ }^{\vee}$ ), mit dem auch Stadens Reisebericht leichter auszuwerten war.

Winckelmann gliedert das zeitgenössische Amerikawissen in elf Abschnitte. Allein drei Kapitel widmen sich der Ethnogenese der Amerikaner. Unter dem Rubrum »Aus was Ort der Welt selbige Einwohner mögen entsprungen seyn? Auch wie und durch was Wege die ersten Menschen und Thiere in die neue Welt kommen? « (ebd.: 55-60) adressiert Winckelmann die seit dem 16. Jahrhundert kontrovers diskutierte Frage, wie sich die Herkunft der >wilden < Völker zur biblischen Genealogie verhalte. ${ }^{18}$ Verworfen werden ältere Erklärungen, der zufolge ein Engel einige Nachkommen Noahs nach Amerika geflogen habe; auch den Seeweg schließt Winckelmann als unwahrscheinlich aus; mit Hugo Grotius und Georg Horn scheint ihm einzig der Landweg möglich, den die Amerikaner entweder über Afrika oder über Grönland gefunden hätten (ebd.: 68-70). Aus der Tatsache, dass die Amerikaner »noch anitzo sehr übel zur Christlichen Kirchen sich accommodiren wollen«, schließt Winckelmann, dass sie keinesfalls von Noahs Sohn Japhet herrühren wie die Europäer, sondern eher von dem »boßhaftigen Cha[m]« (ebd.: 8o), also dem Stammvater der Afrikaner. Damit essentialisiert Winckelmann die kulturelle Differenz zu den Europäern und negiert an anderer Stelle ausdrücklich, dass diese sich noch im paradiesischen >Naturstand <efänden. Stattdessen hält Winckelmann dafür,

die Americaner aus solchen Oertern kommen / woselbsten Policey / Ordnung / Tugend und Kunst im Gebrauch gewesen / massen es wol seyn kan / daß durch Langheit der Zeit solches alles in Vergeß gerathen / und was sie vorhin gewust / aus der Acht gelassen / sich nur mit jhrem blosen Lande befriediget / um Gesetz / Gottesdienst und Ehrbarkeit sich nicht viel bekümmert / sondern mehrentheils nackend ohne alle Schaam / Zucht und und Furcht / gleich dem Vieh / herum gelauffen (ebd.: 90).

Winckelmanns Überlegung markiert gerade die Gegenposition zu Stadens und de Brys Projektion paradiesischer Unschuld: Im Lichte dieser Deutung befinden sich die Amerikaner bereits in einem Zustand der Dekadenz, sind von dem ursprünglichen Zivilisationsstand abgefallen und exemplifizieren damit die Möglichkeit der Regression.

Wie an mehreren Stellen durchklingt, stellt die historische Entwicklung der Amerikaner auch für europäische Christen eine Gefahr dar. Denn überraschend schließt Winckelmann seine Reisekompilation mit der Einsicht,

18 | Siehe dazu Huddleston 1967. 
daß sich die Unholten gegen [Staden] holdseliger / die Rohe und Grausame leutseliger / diejenige / so von keiner Gottselig- und Erbarkeit wusten / sich gegen inn Gottseliger und Aufrichtiger erzeiget hetten / als seine Neben-Christen / und daß inm alle die / bey den grausamen Wilden / ausgestandene Geisselungen / Striemen / eusserste Leibs- und Lebens Gefahr so weh nicht gethan / als inm die Christen / seine Mitgesellen und Bekanten / vermittelst ihrer Schlangen giftigen falschen Zungen sein ehrliches / redliches / unschuldiges Gemüth / Herz und Seele verwundet haben (ebd.: 226f.).

Winckelmann bewegt sich in seiner Schlusspassage ganz auf der Linie der barocken Homiletik, deren Gemeinplätze er aber gleich dreifach beglaubigt, nämlich durch weitere Zitate aus Kolumbus', Johann Moritz' von Nassau und Hans Stadens Reisebüchern. Die kompilierten und kommentierten Anekdoten aus den drei Augenzeugenberichten werden hier hyperbolisch gesteigert, um dann in dem Komparativ zu gipfeln, grausiger und befremdlicher als all dies sei das unchristliche Verhalten der Christen. Anders aber als bei Montaigne, an den diese Passage erinnert, fehlt bei Winckelmann jede Idealisierung der Amerikaner: Konfrontiert wird der Leser mit dem durch zahlreiche Anmerkungen und Nachweise authentisierten Bild eines lasterhaften, grausamen, zudem wohl degenerierten, damit geschichtsfähigen Volks von >Wilden<, deren Verhalten, so die Warnung, doch schlimmer noch ist als das der christlichen Europäer. In diesem Gefüge aus Exotismus und selbstgeißelnder Projektion wird eine hegemoniale Figuration sichtbar, die dem eurozentrischen Denken bis in die Gegenwart eingeschrieben ist: ${ }^{19}$ Winckelmann bestaunt die Monstrosität des Fremden, kartographiert und vermisst es akribisch und entdeckt sich schließlich selbst darin, um seine projektive Aneignung sodann als neutrales Geschichtsurteil zu verkleiden.

\section{"WAS HÖR ICH! " ZUR KURIOSITÄT DES EIGENEN in ERASMUS Franciscis GeSPRÄChSKOMPILATIONEN}

Neben gelehrten Kompilationen wie Winckelmanns Der Americanischen Neuen Welt Beschreibung spielten dialogisch-fiktionale Sammelwerke die größte Rolle bei der Popularisierung von Amerikabeschreibungen vor Beginn des 18. Jahrhunderts. Nach dem Vorbild der humanistischen Convivia und ihrer barocken Wiederentdeckung durch Georg Philipp Harsdörffer erfanden Autoren wie Johannes Praetorius und Eberhard Werner Happel eine fiktionale Rahmenhand-

19 | Sie wirkt, wie Homi Bhabha kritisiert hat, in der Kritischen Theorie des 20. Jahrhunderts nach: Die "shot-reverse-shot strategy of a serial enlightenment" instrumentalisiere das Andere dazu, inre "internal critique of [...] the illusions and delusions of civil society" zu untermauern. Das Andere werde dabei "cited, quoted, framed, illuminated " - letztlich aber erwarte man, "that, in analytical terms, it be always the 'good object of knowledge, the docile body of difference" (Bhabha 1994: 31). 


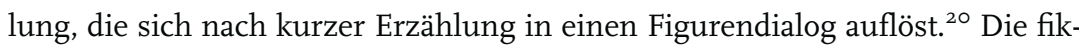
tiven Figuren vermitteln einander enzyklopädisches Wissen aus allen Gebieten, oft in betont disparater-ungeordneter Reihenfolge. Als paradigmatischer Vertreter dieser Gattung kann der Nürnberger Verlagskorrektor Erasmus Francisci gelten, Verfasser von fast 70 selbstständigen, teils voluminösen Publikationen. ${ }^{21}$ Francisci sammelte geographische, ethnographische und historische Informationen aus verschiedenen Reiseberichten, beschränkte sich aber nicht auf amerikanische Sensationen, sondern vermengte Bemerkenswertes aus allen Kontinenten. So wie später Eberhard Werner Happels Thesaurus Exoticorum (1688), der eine ganze > Schatzkammer < persischer, chinesischer, ägyptischer, lybischer, >hottentottischer $<$, brasilianischer, mexikanischer usw. Sittenbeschreibungen aufbot, beschränken sich auch Franciscis exotistische Kompilationen durchaus nicht auf amerikanische Sensationen, sondern vermengen Bemerkenswertes aus allen Kontinenten, um gegen die Pluralität des Anderen das kulturell Eigene abzuwägen. »Zu der vollkommenen Selbst-Erkenntniß«, so Franciscis Maxime entsprechend, gehöre auch die »Erkenntniß andrer Menschen«, »wie nemlich dieselbe in ihrer Weise / Gewonheit / und Bräuchen / gesittet«: »Nimm weg alle Kundschafft fremder Sitten: die Sittsamkeit selbst wird gewißlich / bey uns / nicht gar lang eine Landsassinn bleiben [...]. Kurtz / was uns fehlt / das sehen wir nicht allein / an uns; sondern auch / an andren « (Francisci 1670: fol. [):(] j j). $^{\mathrm{r}}$.

In Erasmus Franciscis früher Kompilation Die Lustige Schau-Bühne (1663) findet sich eine Anekdote aus Jean de Lérys Brasilienreisebericht, die auch Winckelmann mitteilt, dort versehen mit der Marginalie »Ein Indianer fragt / ob die Spanier kein Holz in ihrem Land hetten?« (Winckelmann 1664: 116). Bereits Jahrzehnte zuvor hatte Julius Wilhelm Zincgref sie in seine erfolgreiche Apophthegmata-Sammlung (1626-1631, Wiederauflagen bis 1693) aufgenommen, hier hatte sie den Titel getragen »Indianer achten keines Reichthumbs« (Zincgref 2011: 382 [Nr. 160/2111]). ${ }^{22}$ Francisci legt die Anekdote dem Gastgeber einer klei-

20 | Zum Wirken der Gattungstradition auf Erasmus Francisci siehe Timmermann 1999.

21 | Zu Aspekten der Wissensordnung und -vermittlung bei Francisci siehe Meierhofer 2010: 215-260, bes. 230-236. Zur Amerikadarstellung in Franciscis CEuvre vgl. Ferraris 1995 u. Kramer 1995.

22 | Bei Zincgref lautet das Apophthegma wie folgt: "Ein Indianer sahe ein Frantzösisch Schiff mit Brasilien Holtz laden / fragt derhalben Johannem Lerium, ob kein Holtz bey den Christen wachse / das sie so sehr vnd gefährlich in das Holtz fahren? Lerius antwortet: Ja / es wachst freilich Holtz genug bey vns / aber nicht diser gattung / so braucht man auch dises Holtz nicht zum brennen / sondern zum ferben. Der Indianer fragt weiter: ob man also gar vil haben müste? Lerius antwortete: Es ist bey vns ein einiger Kauffman / der kaufft allein all dises Holtz / der hat mehr roht Tuch / Scheren / Spiegel etc. (dann dergleichen wahr seyn den Indianern am meisten bekant) dann jemahls in dises Land kommen. Der Indianer verwundert sich vnd fragt / ob dann diser Kauffmann auch sterblich were? Dem gab Lerius zur antwort: Er were eben so wol sterblich / als ein anderer Mensch auch. Da fragt er weiters: wer dann nach sei- 
nen Gruppe von Freunden in den Mund, der sie stark erweitert und wie folgt einleitet:

Man beklagt zwar [...] die Hartnäckigkeit so wol dieser West- als der Ost-Indianer / daß sie so gar schwer zu bekehren: aber unsre eigene Unart / Geitz / Uneinigkeit und Spaltungen stehen vielmehr zu beseuftzen [...]. Gar denckwürdig ist die Unterredung / so Franciscus Lerius, laut seiner eigenen Brasilianischen Schiffarths-Beschreibung / mit einem wilden Menschenfresser gehalten. Jetztgenannter Lerius, der in einer Frantzösischen Vestung / die mit diesen Menschenfressern im Bunde war / sich aufhielte [...] ward einsmals von einem Wilden / den er / in seinem Hüttlein besuchte / in guter Verträulichkeit gefragt: Warum sie [...] doch so häuffig von innen das Brasilien-Holtz erhandelten / und einschifften? Wächst / (fragte er) in eurem Lande / kein Holtz auf dem Heerd zu brennen?

Es wächst bey uns Holtzes gnug; antwortete Lerius / aber nicht von solcher Art / wie das eurige ist: bevorab das Brasilien-Holtz / welches die Unsrigen / nicht zum brennen [...] / sondern zum färben mit sich nehmen [...]. Aber / (hebt der Wilde wieder an) habt ihr dann eben eine so grosse Menge deß Holtzes [...] vonnöthen? Freilich / gab Lerius zur Antwort: Denn weil / bey uns / oft ein einiger Kauffmann / mehr Scharlach / oder rotes Tuch [...] besitzt; weder jemaln hieher zu euch gebracht worden: als kan ein so wohlhabender Kauffmann alles Brasilien-Holtz / so in einem Schiff vorhanden / allein kauffen / und verhandeln; deßweden dann nothwendig mehr Schiff damit allhie müssen befrachtet werden.

Was hör ich! sprach der barbarische Mensch; du redest von wunderlichen Dingen! Und nachdem er ein wenig / mit stiller Verwunderung / inne gehalten; hub er an / von neuen zu forschen: Aber / mein! derselbige reiche Handelsmann nun / von dem du mir gesagt; stirbt er dann auch nicht? Ja er stirbt (versetzt Lerius) gleichwie andre Menschen.

Darauf der Wilde ferner [...] von inm forschet: Wer erbet denn die Güter / so ein solcher Sterbender hinterlässt?

Seine Kinder (antwortete Lerius) da er anders welche hat: in Ermangelung deren aber; die Brüder / Schwestern / oder seine nächste Anverwandten.

nem todt sein groß Reichthumb besitze? Sagt Lerius: seine Kinder / oder wann er keine hat / seine nechste Blutsfreundt. Da fieng der Indianer vberlaut an zulachen / vnd sprach: Seyt jhr dann nicht Narren / daß jhr euch mit so grosser leibs vnd lebens gefahr einen so weiten Weg zu Wasser vnd Landt bemühet vnd waget / damit jhr euren Kindern vnd Freunden groß Reichthumb verlassen möcht / vertrawet jhr nicht / daß eben das Erdrich / welches euch vnd eure Vorfahren vor euch ernehret hat / auch eure Kinder vnd Nachkömling nach euch ernehren vnd erhalten könne?" - Francisci zitiert Léry freilich sehr häufig und es gibt kein Indiz dafür, dass er das Gespräch zwischen Léry und dem Indianer Zincgrefs Apophthegmata entnommen hat. Dennoch belegt der dortige Abdruck, wie weit Lérys Reisebericht in Deutschland verbreitet war, sowie dass die kulturkritischen Spitzen, die sich dort finden, von den Zeitgenossen durchaus erkannt wurden. Zur Léry-Rezeption in der englischen und französischen Aufklärung siehe Lestringant 1993. 
Wie er diß gesagt; spricht der alte Barbar wieder zu inm: Hieraus kan ich leichtlich abnehmen / daß ihr Frantzosen sehr grosse Thoren seyn müst. Denn was ist es nöthig / daß ihr so grosse Mühe auf euch nehmet / über Meer schiffet / und [...] so viel Gefahr und Ungemach erleidet; damit ihr euren Kindern und nächsten Erben Güter erwerbet? Ist die Erde / welche uns bißher hat ernährt / nicht gnug / auch sie zu ernähren? Wir zwar haben auch unsre Kinder und Verwandten; und lieben dieselbe / wie du siehest / gantz inbrünstig: weil wir aber das Vertrauen haben / es werde das Land / so uns erhalten / auch ihnen ihren Unterhalt schaffen; sind wir im übrigen unbekümmert / und wohl zu frieden.

Was dunckt den Herren / um diese Worte? solten sie nicht einem tugendhafften Heyden / will geschweigen einem Christen / ein Färbchen abjagen? (Francisci 1663: 1008f.)

Der Tadel des >alten Barbaren< trägt unverkennbar noch die Züge einer jahrhundertealten christlichen Gesellschaftskritik: Seine Worte richten sich gegen den Reichtum des Kaufmanns, der sich, wenn er ganze Schiffsladungen von Holz nur zum Einfärben verbraucht, der Sünden der Verschwendung und Gefallsucht schuldig macht. Das implizite Memento mori der dritten Frage, ob der Kaufmann nicht dereinst sterben werde, entlarvt sein Handeln als gottlose Weltvernarrtheit, als Ausdruck von Habgier und Ausschweifung. Doch der indianische Weise missbilligt nicht nur das Handeln des einzelnen Kaufmanns, sondern bezieht seine Kritik auf eine ganze Wirtschaftsform, die er als schlechthin unökonomisch verurteilt: Unter Aufwendung größter Kräfte und Inkaufnahme enormer Risiken verschwenden die Franzosen natürliche Güter, die ihren Nachkommen ohnehin zur Verfügung stehen würden. Der Spott des Indianers ergießt sich über das Prinzip der Güterakkumulation für künftige Generationen, die des Erbes eigentlich nicht bedürften - sie könnten sich doch schlicht der Mittel bedienen, welche die Natur ihnen zu ihren Lebzeiten zur Verfügung stellen wird! Der Indianer verachtet nicht nur den Reichtum an sich, sondern verurteilt mit der vierten Frage auch das Konzept der wirtschaftlichen Zukunftsvorsorge überhaupt, das aus seiner Sicht von mangelndem »Vertrauen « in die natürliche Fortdauer der Güter zeugt. Dass die Indianer »unbekümmert / und wohl zu frieden « sind (lies: und die Franzosen nicht), erklärt sich aus dem ökonomischen Prinzip des zuversichtlichen In-den-Tag-hinein-Lebens, das man in Europa verlernt hat.

Bedeutend ist die Anekdote aber nicht nur aufgrund des Gegenstands der Kritik, sondern auch wegen der Form, in der Léry und Francisci sie vortragen lassen. Der europäisch-brasilianische Dialog nämlich wird hier zum wirkungsvollen Mittel einer Art >sokratischer< Persuasion. Der französische Leser identifiziert sich mit der Figur >Léry<, gegen den der exotisierte >Wilde< zunächst als Inbegriff des Anderen erscheint. Durch die kritischen Nachfragen seines Gegenübers gerät aber die Identifikationsfigur sukzessive in Rechtfertigungszwang und ist am Schluss in seinen Überzeugungen erschüttert - was sich auf den Leser und seine Sympathien übertragen soll. Besonderes Gewicht kommt dabei der Inszenierung der Indianerfigur zu: Der »alte Barbar« reagiert auf Lérys 
Schilderungen zunächst mit intuitivem Erstaunen (interjektiv: »Was hör ich!«), dann mit bedächtiger Prüfung des Gehörten (»Und nachdem er ein wenig / mit stiller Verwunderung / inne gehalten«), schließlich mit resoluter Ablehnung (»Hieraus kan ich leichtlich abnehmen / daß ihr Frantzosen sehr grosse Thoren seyn müst«). Die Reaktionsphasen des Indianers - vom Nichtverstehen über das Bedenken zum Urteilen - verhalten sich dabei spiegelverkehrt zum selbstreflexiven Erkenntnisprozess Lérys und mithin des Lesers. Mag der Leser am Anfang von der Sinnhaftigkeit des französischen Holzhandels überzeugt sein und die Kritik des Indianers nicht begreifen, muss er am Ende einsehen, wie Recht der >Barbar < mit seinen Einwänden hat. In diesem Erstaunen und Spotten über das Eigene, in dem Verrätseln des Selbstverständlichen gründet sich die Wirkmächtigkeit der Passage, die Francisci hier ja lediglich aufnimmt, nicht erfunden hat. Sie verkehrt das Überlegenheitsgefühl des Europäers beim Anblick der brasilianischen Kultur und imaginiert, mit welcher Fassungslosigkeit ein Brasilianer auf die europäische Kultur blicken muss.

\section{Die neue als die alte Welt: AufkLÄrerische Inversionen}

Anders als sein Zeitgenosse Winckelmann, für den die amerikanischen $>$ Unsitten auf frühgeschichtliche Zivilisationsregression zurückzuführen waren, schließt Francisci an die Paradiesprojektionen der frühen Reiseberichte an, die er in seiner Vorrede ausdeutet. Stellen wie die vorliegende antizipieren die frühaufklärerische Ursprungsimagination eines vorzivilisatorischen Naturstands, wie sie etwa in Louis-Armand Baron de Lahontans skandalösen Nouveaux Voyages dans l'Amerique Septentrionale (1703) entwickelt wird. ${ }^{23}$ Im dritten Band seiner Reisebeschreibung schildert Lahontan einen Dialog zwischen dem Franzosen Lahontan (wie bei Léry ein Alter Ego des Autors) und dem Huronen Adario. Diskutiert werden die jeweiligen Vorzüge der beiden Kulturen, wobei Lahontan seinem sedlen Wilden sche Zivilisation und die christliche Religion in den Mund legt. Wie Lérys kurzes brasilianisches Gespräch vertauscht auch Lahontans fiktionaler Huronendialog Subjekt und Objekt der ethnographischen Betrachtung: Im unbestechlichen Blick des klugen Huronen erscheint plötzlich Europa, nicht Amerika als rätselhaft und erklärungsbedürftig. ${ }^{24}$ Damit beeinflusste Lahontan spätere Idealisierungen des ständelosen Naturzustands von Voltaires L'Ingénu (1767) bis zu

23 | Vgl. Detering 2017: 490f.

24 | In diesem Sinne postuliert auch Kohl (1983: 76), Lahontans Nouveaux Voyages bildeten den "wenn schon nicht historische[n], so doch logische[n] Schlußpunkt jener Form ethnologischer Betrachtungsweise, die sich der ethnographischen Beobachtungen vornehmlich zu dem Zweck bedient, auf ihrer Grundlage ein Raster zu entwickeln, durch das kritisch zurückgeblickt werden kann auf die eigene Kultur, jener Form der Ethnographie mithin, deren eigentlicher Gegenstand nicht so sehr die fremde, als vielmehr die eigene Gesellschaft bildet». 
Diderots Supplément au voyage de Bougainville (1772, veröffentlicht 1796). ${ }^{25}$ Auch dort pries man die Ursprünglichkeit der indigenen Völker, kehrte jedoch die Metaphorik weltgeschichtlicher Altersstufen um. Diderot etwa betont statt der Entstehungspräzedenz den Entwicklungsstand einer Kultur. Amerika kann so als jugendlich, weil angeblich kaum gewachsen, Europa hingegen als alt und erfahren, weil stark entwickelt gelten: »Le Tahitien touche à l'origine du monde«, postuliert Diderot 1772 in seinem Supplément au voyage de Bougainville (postum 1796 veröffentlicht), »et l'Européen touche à sa vieillesse ${ }^{26}$ (zit. n. Steinkamp 2003: 159). ${ }^{27}$

Doch schon vor der radikalen Satire Lahontans finden sich Belege für diese Amerikadeutungen, die sich auch der barocken Kompilatorik verdanken. Bereits 1697 dichtet der Hamburger Gelehrte Christian Wernicke in einem Alexandriner-Epigramm Auf die Eroberung von Mexico:

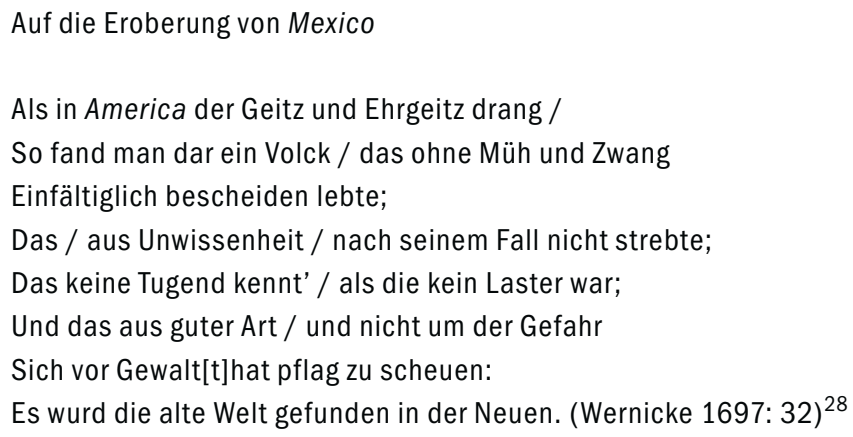

Die räumlich-kulturelle Distanz zwischen den europäischen Reisenden und den Amerikanern erhält bei Wernicke eine historische Tiefendimension: Im Spiegel der neuentdeckten Fremdheit sieht er das vergangene Eigene. Die epistemologischen Zuschreibungen von >alt< und >neu<-das altbekannte Europa versus das neuentdeckte Amerika - werden invertiert und geschichtsphilosophisch umgedeutet: Alt, weil einfältig, unschuldig, vorgeschichtlich, erscheint nun Amerika; der neue, weil historisch gewachsene, ursprungsentfernte, später: moderne Kontinent wird Europa.

25 | Zu Lahontans Dialogues curieux siehe Dölling 2013, Ellingson 2001: 65-76, und Funke 2003.

26 | "Der Tahitianer steht dem Ursprung der Welt nahe [...] und der Europäer ihrem Greisenalter" (Übers. N.D.).

27 | Bereits José de Acosta und einige seiner Zeitgenossen hatten die Parameter ralt und neur im Zusammenhang mit der Amerikawahrnehmung problematisiert (vgl. Burghartz 2004).

28 | Wernicke hat das Gedicht für Neuauflagen seiner Epigrammata 1701 und 1704 stark überarbeitet und 1704 sogar noch durch einen (etwas dunklen) 'Gegensatz، erweitert, der die Zerstörung der amerikanischen Unschuld beklagt; siehe dazu ausführlich Klaffke 2000: 70-84; vgl. auch Neuber 1991: 54f. 


\section{LITERATUR}

Acosta, José de (1598): Historie Naturael ende Morael van de Westersche Indien. Waer inne ghehandelt wordt van de merckelijcktse dinghen des Hemels, Elementen, Metalen/Planten ende Ghedierten van dien. Übers. v. Johann Huygen van Linschoten. Amsterdam.

Ders./Jansz, Barent (1601): Neundter vnd Letzter Theil Americæ [...] Alles auffs trewlichste aus Niederländischer Beschreibung Iohan. Hugen von Lintschotten. Übers. v. Johann Humberger. Frankfurt a.M.

Arend, Stefanie (2006): Interkulturelle Begegnungen. Europäer und Kannibalen in der (Reise-)Literatur der Frühen Neuzeit. Von Kolumbus bis Wezel. In: Hans-Jürgen Lüsebrink (Hg.): Das Europa der Aufklärung und die außereuropäische koloniale Welt. Göttingen, S. 326-355.

Atkinson, Geoffroy (1935): Les Nouveaux Horizons de la Renaissance Française. Paris. Avity, Pierre d' (1638): Archontologia Cosmica, Sive Imperiorum, Regnorum, Principatuum, Rerumque Publicarum Omnium Per Totum Terrarum Orbem Commentarii Luculentissimi. Frankfurt a.M.

Berlin, Isaiah (1991): Alleged Relativism in Eighteenth-Century European Thought [1980]. In: Ders.: The Crooked Timber of Humanity. Chapters in the History of Ideas. Hg. v. Henry Hardy. London, S. 70-91.

Bhabha, Homi K. (1994): The Location of Culture. London.

Bitterli, Urs (1976): Die 'Wilden، und die 'Zivilisierten`. Grundzüge einer Geistes- und Kulturgeschichte der europäisch-überseeischen Begegnung. München.

Blumenberg, Hans (1966): Die Legitimität der Neuzeit. Frankfurt a.M.

Böning, Holger (2011): Der Kalender im Mediensystem des 17. Jahrhunderts. In: Peter Heßelmann (Hg.): Grimmelshausen als Kalenderschriftsteller und die zeitgenössische Kalenderliteratur. Bern u.a., S. 13-33.

Burghartz, Susanna (2004): Alt, neu oder jung? Zur Neuheit der ,Neuen Welt. In: Achatz von Müller/Jürgen von Ungern-Sternberg (Hg.): Die Wahrnehmung des Neuen in Antike und Renaissance. Berlin, S. 182-200.

Christadler, Maike (2004): Die Sammlung zur Schau gestellt. Die Titelblätter der America-Serie. In: Susanna Burghartz (Hg.): Inszenierte Welten. Staging New Worlds. Die west- und ostindischen Reisen der Verleger de Bry, 1590-1630. Basel, S. 47-89.

Conrad, Sebastian/Randeria, Shalini (2002): Geteilte Geschichten - Europa in einer postkolonialen Welt. In: Dies. (Hg.): Jenseits des Eurozentrismus. Postkoloniale Perspektiven in den Geschichts- und Kulturwissenschaften. Frankfurt a.M./New York, S. 9-50.

Detering, Nicolas (2017): Krise und Kontinent. Die Entstehung der deutschen EuropaLiteratur in der Frühen Neuzeit. Köln/Weimar/Wien.

Dölling, Corinne M. (2013): 'Mes amis sauvages`. Die Reiseberichte Louis-Armand de Lahontans als Dokumente der Frühaufklärung. München.

Ellingson, Ter (2001): The Myth of the Noble Savage. Berkeley/Los Angeles/London. Elliott, John H. (1970): The Old World and the New, 1492-1650. Cambridge.

Enders, Angela (1993): Die Legende von der "Neuen Welt". Montaigne und die ,littérature géographique، im Frankreich des 16. Jahrhunderts. Tübingen. 
Ferraris, Francesca (1995): Neue Welt und literarische Kuriositätensammlungen des 17. Jahrhunderts: Erasmus Francisci (1627-1694) und Eberhard Werner Happel. In: Karl Kohut (Hg.): Von der Weltkarte zum Kuriositätenkabinett. Amerika im deutschen Humanismus und Barock. Frankfurt a.M., S. 93-108.

Francisci, Erasmus (1663): Die lustige Schau-Bühne von allerhand Curiositäten: darauf Viel nachdenckliche Sachen/sonderbare Erfindungen/merckwürdige Geschichte/Sinn- und Lehr-reiche Discursen / auch zuweilen anmuthige Schertz-Reden und Erzehlungen/fürgestellet werden. Nürnberg.

Ders. (1670): Neu-polirter Geschicht- Kunst- und Sitten-Spiegel ausländischer Völcker/fürnemlich Der Sineser/Japaner/Indostaner/Jabaner/Malaber/Peguaner [...] und theils anderer Nationen mehr: welcher/in sechs Büchern/sechserley Gestalten weiset [...]. Nürnberg.

Freytag, Peter (1677): Alter und Neuer Cosmographischer Kalender [...] Auf das Jahr [1678]. Nürnberg.

Frübis, Hildegard (2008): Zivilisationskritik im Zeichen des Kannibalismus. Jean de Lérys Histoire d'un voyage fait en la terre du Brésil in Bild und Text. In: Ulrike Ilg (Hg.): Text und Bild in Reiseberichten des 16. Jahrhunderts. Westliche Zeugnisse über Amerika und das Osmanische Reich. Venedig, S. 193-213.

Funke, Hans-Günter (2003): Das interkulturelle Streitgespräch zwischen Europäer und "Wildem" als Medium aufklärerischer Zivilisationskritik. La Hontans Dialogues curieux entre l'auteur et un sauvage (1703). In: Gabriele Vickermann-Ribémont/ Dietmar Rieger (Hg.): Dialog und Dialogizität im Zeichen der Aufklärung. Tübingen, S. 69-95.

Grafton, Anthony (1992): New Worlds, Ancient Texts. The Power of Tradition and the Shock of Discovery. Cambridge/London.

Greve, Anna (2004): Die Konstruktion Amerikas. Bilderpolitik in den 'Grands voyages aus der Werkstatt de Bry. Köln u.a.

Grimmelshausen, Johann Jakob Christoffel von (2009): Simplicianischer Jahreskalender. Faksimiledruck der vier Kalenderjahrgänge. Hg. v. Klaus Matthäus/Klaus-Dieter Herbst. Erlangen/Jena.

Groesen, Michiel van (2008): The Representations of the Overseas World in the De Bry Collection of Voyages (1590-1634). Leiden u.a.

Hampton, Timothy (1997): The Subject of America: History and Alterity in Montaigne's Des coches. In: Romanic Review 88, S. 203-27.

Happel, Eberhard Werner (1688): Mundi Mirabilis Tripartiti, Oder Wunderbaren Welt / in einer kurtzen Cosmographia fürgestellet/Anderer Theil/Welcher handelt Von den Menschen auf der Welt/von ihren Dignitäten/Potentaten/Religionen/Estats-Maximen/Macht/Intraden/Krieg-Art/Waffen/Policey [...]. Ulm.

Harbsmeier, Michael (1994): Wilde Völkerkunde. Andere Welten in deutschen Reiseberichten der Frühen Neuzeit. Frankfurt a.M./ New York.

Hazard, Paul (1935): La Crise de la conscience européenne (1680-1715). Paris.

Holdenried, Michaela (2004): Künstliche Horizonte. Alterität in literarischen Repräsentationen Südamerikas. Berlin.

Huddleston, Lee E. (1967): Origins of the American Indians. European Concepts, 14921729. Austin. 
Itzapolka, Coran [d. i. Peter Freytag?] (1675): Alter und Neuer Americanischer Schifffahrten/Sitten/Trachten/Grausamkeiten und Landschafften Calender Auf das Jahr [...] [1676]. Nürnberg.

Kenny, Neil (2004): The Uses of Curiosity on Early Modern France and Germany. Oxford Kiening, Christian (2006): Das wilde Subjekt. Kleine Poetik der neuen Welt. Göttingen.

Klaffke, Andreas (2000): "Es sey die alte Welt gefunden in der Neuen«. Amerika in der deutschen Lyrik der Frühen Neuzeit. Marburg.

Kohl, Karl-Heinz (1983): Entzauberter Blick. Das Bild vom Guten Wilden und die Erfahrung der Zivilisation. Frankfurt a.M./Paris.

Kramer, Roswitha (1995): Die Neue Welt als Lustgarten. Amerika im Werk von Erasmus Francisci. In: Karl Kohut (Hg.): Von der Weltkarte zum Kuriositätenkabinett. Amerika im deutschen Humanismus und Barock. Frankfurt a.M., S. 108-152.

Léry, Jean de (1578): Histoire d'vn voyage faict en la terre dv bresil, avtrement dite Amerique. [...] Paris.

Lestringant, Frank (1990): Le Huguenot et le Sauvage. L'Amérique et la Controverse Coloniale, en France, au Temps de Guerres de Religion (1555-1589). Paris.

Ders. (1993): The Philosopher's Breviary: Jean de Léry in the Enlightenment. In: Stephen Greenblatt (Hg.): New World Encounters. Berkeley, S. 127-139.

Ders. (1999): Jean de Léry ou l'invention du sauvage. Essai sur L'Histoire d'un voyage faict en la terre du Brésil. Paris.

Mason, Peter (1994): Classical Ethnography and its Influence on the European Perception of the Peoples of the New World. In: Wolfgang Haase/Reinhold Meyer (Hg.): The Classical Tradition and the Americas. Bd. 1: European Images of the Americas and the Classical Tradition. Berlin/ New York, S. 135-172.

Meierhofer, Christian (2010): Alles neu unter der Sonne. Das Sammelschrifttum der Frühen Neuzeit und die Entstehung der Nachricht. Würzburg.

Montanus, Arnaldus/Dapper, Olfert (1673): Die Unbekante Neue Welt/oder Beschreibung des Welt-teils Amerika, und des Sud-Landes: Darinnen vom Vhrsprunge der Ameriker und Sudländer/und von den gedenckwürdigen Reysen der Europer darnach zu [...] ausführlich gehandelt wird. Übers. v. Johann Christoph Beer. Amsterdam.

Neuber, Wolfgang (1991): Fremde Welt im europäischen Horizont. Zur Topik der deutschen Amerika-Reiseberichte der Frühen Neuzeit. Berlin.

Pagden, Anthony (1993): European Encounters with the New World. From Renaissance to Romanticism. New Haven/London.

Perplies, Helge (2017): Inventio et repraesentatio Americae. Die India OccidentalisSammlung aus der Werkstatt de Bry. Heidelberg.

Seth, Vanita (2010): Europe's Indians. Producing Racial Difference, 1500-1900. Durham.

Staden, Hans (1557): Warhaftig Historia vnd beschreibung eyner Landtschafft der Wilden, Nacketen, Grimmigen Menschfresser Leuthen, in der Newenwelt America gelegen. Marburg.

Ders./ Léry, Jean de (1593): Dritte Buch Americæ, Darinn Brasilia durch Johann Staden [...] auß eigener erfahrung in Teutsch beschrieben. Item Historia der Schiffart Ioannis Lerij in Brasilien [...]. Frankfurt a.M. 
Steinkamp, Volker (2003): L’Europe éclairée. Das Europa-Bild der französischen Aufklärung. Frankfurt a.M.

Stöffler, Isaac Conrad (1673): Alter und Neuer Cosmographischer Calender/Das ist: Asiatisch-Africanisch-Americanisch-Europäischer Königreich/Länder/Städte/ Schlösser/Vestungen/Sitten/Trachten und Geschichten Beschreibung. [...] Auf das Jahr [...] [1674]. Nürnberg.

Strauss, Walter L. (Hg.; 1980): The Illustrated Bartsch. Bd. 10: Sixteenth Century German Artists. Albrecht Dürer. New York.

Talbot, Ann (2010): "The Great Ocean of Knowledge". The Influence of Travel Literature on the Work of John Locke. Leiden/Boston.

Timmermann, Ina (1999): "Löbliche Conversation“ als ,Einübung ins Räsonnement،. Das Gespräch als Ziel und Funktion barocker Erzählsammlungen am Beispiel der Lustigen Schau-Bühne von allerhand Curiositäten des Erasmus Francisci (1627-1694). In: Simpliciana 21, S. 15-40.

Watson, Kelly L. (2015): Insatiable Appetites. Imperial Encounters with Cannibals in the North Atlantic World. New York.

Weinberg, Bernard: (1968): Montaigne's readings for Des Cannibales. In: George Bernard Daniel (Hg.): Renaissance and Other Studies in Honor of William Leon Wiley. Chapel Hill, S. 264-279.

Wernicke, Christian (1697): Uberschriffte Oder Epigrammata, In kurtzen Satyren, Kurtzen Lob-Reden und Kurtzen Sitten-Lehren bestehend [...]. Amsterdam.

Winckelmann, Hans Just (1664): Der Americanischen NEUEN WELT Beschreibung/Darinnen deren Erfindung/Lager/Natur/Eigenschaft/Sitten/Barbarey/und unerhörte Grausamkeit der Einwohner [...]; Beneben einer wunderbaren Schifffart und ReiseBeschreibung nach Brasilien Hans von Staden [...] Mit vielen nachdenklichen Fragen und nothwendigen Figuren ausgezieret und zusammen getragen. Oldenburg.

Wüthrich, Lucas Heinrich (1993): Das druckgraphische Werk von Matthaeus Merian d.Ä. Bd. 3. Die großen Buchpublikationen I. Hamburg.

Zincgref, Julius Wilhelm (2011): Apophthegmata teutsch. Gesammelte Schriften Bd. IV. Hg. v. Theodor Verweyen/Dieter Mertens/Werner Wilhelm Schnabel. 1. Teilbd. Text. Berlin/Boston. 